\title{
Comments on: Single and two-stage cross-sectional and time series benchmarking procedures for small area estimation
}

\author{
Neung Soo Ha • Partha Lahiri
}

Published online: 21 October 2014

(C) Sociedad de Estadística e Investigación Operativa 2014

We would like to congratulate the authors for providing an excellent critical review of the growing body of literature on benchmarking in small area estimation and illustrating how two-stage benchmarking procedure works for a hierarchical time series model to estimate monthly unemployment rates for the US Census Divisions and states. Although we generally agree with them, we feel that a few points need further explanation and discussion.

As authors explain, benchmarking is an exact property that is relevant to a large area covering a group of small areas and ensures small area estimates, when appropriately aggregated, to yield a specified reliable estimate or a known quantity for the corresponding large area. The authors also discuss the issue of retaining the important design-consistency property for benchmarked small area estimators. Designconsistency is an asymptotic property of a survey estimator under which the estimator approaches to the finite population parameter of interest as the sample size grows.

One could naturally question the relevance of design-consistency for estimators in small areas because within area sample sizes are rarely large. Without the in-built design-consistency property, small area estimators contain an undesirable characteristic that they will not be close to the corresponding parameters of interest for areas for which additional large samples become available. Standard direct area estimators are generally design-consistent under certain regularity conditions and are internally benchmarked. However, it is now widely accepted that direct estimators should not be

This comment refers to the invited paper available at doi:10.1007/s11749-014-0398-y.

N. S. Ha

SAMSI, Durham, NC, USA

P. Lahiri $(\bowtie)$

JPSM, University of Maryland, College Park, USA

e-mail: plahiri@survey.umd.edu 
used for estimating small area parameters because of their instability in terms of high variability. Thus, implicit or explicit models along with sophisticated model-based statistical estimation procedures must be used to link primary source of data with various supplementary information from administrative and census records to reduce variability in small area estimators. However, such an approach may not necessarily yield estimators that are design-consistent and/or benchmarked. This has generated a large number of papers, especially in the last few years, which the authors have critically reviewed in this paper.

We would like to further discuss about raking or ratio benchmarking. Raking is a commonly used benchmarking technique among survey samplers and it is possibly the first method used to benchmark small area model-based estimates; see Fay and Herriot (1979). Authors have discussed differences between raked estimator, given by (2), and additive benchmarked estimator, given by (3). The additive benchmarked small area estimators are expected to be design-consistent for self-weighing design under certain regularity conditions. They are also expected to perform better than the corresponding raked estimator in terms of the mean squared error (MSE) criterion since they can generated by minimizing the MSE subject to the benchmarking constraint. In contrast, the authors have rightly argued that the ratio benchmarking (2) is not a special case of their constrained optimization method.

We first demonstrate that a commonly used raked estimator can be obtained using a different loss function in the following constrained optimization process:

Suppose our objective is to minimize $E\left[\sum_{k=1}^{D} b_{k}\left\{\hat{Y}_{k}\left(\ln \hat{Y}_{k}-\ln Y_{k}\right)-\left(\hat{Y}_{k}-Y_{k}\right)\right\}\right]$ with respect to $\hat{Y}=\left(\hat{Y}_{1}, \ldots, \hat{Y}_{D}\right)^{\prime}$ subject to the benchmarking constraint $\sum_{k=1}^{D} b_{k} \hat{Y}_{k}=\sum_{k=1}^{D} b_{k} y_{k}$. Note the connection between the loss function used in this constrained minimization problem and one of the distance functions given in Deville and Sarndal (1992) in the context of generating calibration estimators for large areas. It can be easily seen that this constrained minimization procedure yields the following estimator: $\hat{Y}_{d ; R}=\exp \left(a_{d}\right) \frac{\sum_{k=1}^{D} b_{k} y_{k}}{\sum_{k=1}^{D} b_{k} \exp \left(a_{k}\right)}$, where $a_{d}=E\left(\ln Y_{d} \mid\right.$ data) under an appropriate mixed small area model. This estimator is essentially a variation of the estimator proposed in Fay and Herriot (1979). While this particular raked small area estimator and (2) of the paper are expected to be design-consistent when sample sizes for all areas grow under certain regularity conditions, they cease to possess the design-consistency property in some important cases, such as when sample sizes of some of the areas are bounded. But the problem can be rectified if $b_{k}$ in the loss function is replaced by the weight $\phi_{k}$ used to generate the additive benchmarked estimator (3). It can be shown that the resulting generalized raked estimator is obtained as $\hat{Y}_{d ; G R}=\exp \left(a_{d}\right) R_{d}$, where $R_{d}$ is an area-specific general raking factor given by $R_{d}=\exp \left(-\gamma b_{d} / \phi_{d}\right)$ with $\gamma$ obtained by solving $\sum_{k=1}^{D} b_{k} \exp \left(a_{k}\right) \exp \left(-\gamma b_{k} / \phi_{k}\right)=\sum_{k=1}^{D} b_{k} y_{k}$.

As mentioned before, the benchmarked estimator given by (3) is design-consistent for self-weighing designs. Then a follow-up question is: how do we obtain benchmarked small area model-based estimators that are also design-consistent for a general design? The self-benchmarked estimator of You and Rao (2002) mentioned in the paper provides a partial solution to the problem, but their working model is a nested error regression model, which may not be applicable for other small area situations. 
How does one extend the self-benchmarking idea for complex mixed small area models? One possibility is to extend the idea of self-benchmarking by suitably estimating parameters of the mixed model used in the general design-consistent estimator proposed by Jiang and Lahiri (2006a). Another possibility is to replace the BLUP in (3) of the paper or the best predictor $a_{k}$ in the generalized raked estimator above by a designconsistent estimator such as the ones proposed by Jiang and Lahiri (2006a, b), Lahiri and Mukherjee (2007). The suggested approaches need a thorough investigation.

While benchmarking has been traditionally employed by survey organizations primarily to achieve data consistency, there is a lingering question if this also reduces systematic area-specific biases of model-based small area estimators, especially when the model is grossly misspecified. In this respect we find Remark 4 of the paper interesting and would like to elaborate further on the points raised by the authors. The ratio benchmarking, as described by (2), multiplies model-based estimates of all small areas by the same factor; thus, unless there is no systematic area-specific bias in the modelbased estimators, it is highly unlikely that the single multiplicative factor will reduce any area-specific systematic bias. The adjustments proposed in the additive benchmarking (3) and the generalized raking described earlier are area-specific. However, unless the area-specific systematic biases and the overall bias are in the same direction and area-specific bias is captured by the adjustment factors such as $\lambda_{d}$ in (3), these kinds of cross-sectional benchmarking will hardy reduce the systematic area-specific bias.

The time series benchmarking seems to be more effective in reducing area-specific biases of model-based estimators since it attempts to capture the area-specific bias by exploiting long time series data. Having said that, if it is possible to identify some factors that could explain area-specific systematic bias, it may be possible to reduce it using cross-section benchmarking. In fact, in some applications, this has been tried. For example, Fay and Herriot (1979) considered a two-way benchmarking to make small area estimates to agree with the reliable direct estimates of the margins in a twoway contingency table. In the US Small Area Income and Poverty Estimates (SAIPE) program, hierarchical cross-sectional benchmarking is employed to make small area estimates for counties benchmarked to their corresponding state estimates and the state estimates benchmarked to the national level direct estimate. Similar strategy has been employed in implementing poverty mapping problem in Chile; see Cordero et al. (2014). Suitable cross-sectional benchmarking along with time series benchmarking is yet another option to be explored. These are all indeed complex benchmarking problems and a simple method to achieve this goal is warranted. Recently proposed general benchmarking method of Ha (2013) may be explored in this respect. As the authors note, the important problem of mean squared estimation of benchmarked small area estimators has received less attention. The recently proposed parametric bootstrap method of Chatterjee and Lahiri (2013) is promising in this respect, but needs further investigation.

The authors have nicely tied the recent benchmarking papers dealing with quadratic benchmarking constraints with the adjusted Bayes empirical Bayes idea of Lahiri (1990). We would like to comment that the basic idea of quadratic benchmarking in small area estimation can be attributed to Louis (1984) who proposed constrained Bayes and empirical Bayes estimators to solve the overshrinking problem associated 
with the standard Bayes and empirical Bayes estimators. He used the Lagrange's method of undermined multipliers that minimizes the posterior Bayes risk subject to match the first two moments of the ensemble of small area estimates with those of the Bayes estimate of the histogram of true small area means. Ghosh (1992) extended this idea in a general setting. To make such constrained Bayes or empirical Bayes method robust, one may choose a design-based estimate of the variability of the small area parameters since this continues to be reliable as long as there are enough number of small areas.

We would like to say that benchmarking, if properly done, is desirable. It maintains data consistency and, in addition, has the potential to reduce the biases in small area estimators. We feel that it is always a good practice to examine both unbenchmarked and benchmarked estimates for any unnatural behavior (e.g., estimates going out of admissible ranges). In case of any peculiar pattern in the estimates, one may consider revising the working model and/or the statistical estimation method used.

To summarize, benchmarking has been a very active area of research in small area estimation for the last couple of decades. The elegant exposition given by the authors will definitely stimulate further research in this important research area.

Acknowledgments The second authors research was supported in part by the National Science Foundation Grant Number SES-085100.

\section{References}

Casas-Cordero C, Encina J, Lahiri P (2014) Poverty mapping for the Chilean municipalities, unpublished manuscript

Chatterjee A, Lahiri P (2013) A simple computational method for estimating mean squared prediction error in general small-area model. In: Proceedings of the SAE 2013, Bangkok, http://pioneer.chula.ac.th/ sjirapha/SAE2013/Resampling

Deville JC, Sarndal CE (1992) Calibration estimators in survey sampling. J Am Stat Assoc 87:376-382

Fay RE, Herriot RA (1979) Estimates of income for small places: an application of James-Stein procedures to census data. J Am Stat Assoc 74:269-277

Ghosh M (1992) Constrained Bayes estimation with applications. J Am Stat Assoc 87:533-540

Ha N (2013) Hierarchical Bayesian estimation of small area means using complex survey data. PhD dissertation, University of Maryland, College Park

Jiang J, Lahiri P (2006a) Estimation of finite population domain means - a model-assisted empirical best prediction approach. J Am Stat Assoc 101:301-311

Jiang J, Lahiri P (2006b) Mixed model prediction and small area estimation. Editor's invited discussion paper. Test 15(1):1-96

Lahiri P (1990) “Adjusted" Bayes and empirical Bayes estimation in finite population sampling. Sankhya B 52:50-66

Lahiri P, Mukherjee K (2007) Hierarchical Bayes estimation of small area means under generalized linear models and design consistency. Ann Stat 35:724-737

Louis TA (1984) Estimating a population of parameter values using Bayes and empirical Bayes methods. J Am Stat Assoc 79:393-398

You Y, Rao JNK (2002) A pseudo-empirical best linear unbiased prediction approach to small area estimation using survey weights. Can J Stat 30(3):431-439 